\title{
Fingerprinting Proteins Coupled with Polymers by Mass Spectrometry: Investigation of Polyethylene Glycol-Conjugated Superoxide Dismutase
}

\author{
Swapan K. Chowdhury, Muriël Doleman, and David Johnston \\ Research Division, Sanofi-Winthrop, Inc., Collegeville, Pennsylvania, USA
}

Matrix-assisted laser desorption-ionization (MALDI) mass spectrometry was investigated as a method for the rapid determination of the extent of polymer coupling in polyethylene glycol- (PEG) conjugated superoxide dismutase (SOD). PEG-conjugated SOD, an antioxidant with an extended in vivo circulation lifetime compared to that of superoxide dismutase, is being evaluated as an effective therapeutic agent for the treatment of injuries and arthritis. The mass spectra of a standard batch of PEG-conjugated bovine SOD showed the presence of identifiable and well resolved peaks that correspond to 0-7 PEG molecules attached to bovine SOD. The area of each of the peaks provides a determination of the amount of PEG-conjugated SOD with a given number of bound PEG groups. SOD is a noncovalent dimer of two identical subunits that dissociates in MALDI. The information obtained in the mass spectra thus corresponds to a monomer of SOD. Each SOD monomer contains 10 lysines, which are the sites of PEG-conjugation. Multiple MALDI determinations of two batches of samples indicated good reproducibility for routine determination of the extent of polymer content. The amount of PEG-conjugated SOD that contained a given number of PEG molecules, determined by MALDI, was compared with the value deduced from the amount of PEG-conjugation at each attachment site measured by a peptide mapping method. Agreement between the data obtained in the two techniques (MALDI and peptide mapping) indicates that MALDI may be used to obtain quantitative information on PEG-conjugated SOD to determine the amounts of PEG-conjugated protein each with a different number of PEG groups attached. Measurement of several batches of samples stored at a higher temperature showed a lower extent of PEG-conjugation in PEG-conjugated SOD. This reduction in the PEG content resulted from the PEG-deconjugation of PEG-conjugated SOD at a higher temperature. Thus, MALDI can be used to examine the stability of PEG-conjugated SOD. The high sensitivity, relatively straightforward data interpretation, speed of analyses, and good reproducibility in measurements make this technique a useful analytical tool for fingerprinting PEG-conjugated SOD as well as potentially other polymer-conjugated proteins. (J Am Soc Mass Spectrom 1995, 6, 478-487)

$\mathrm{U}$ se of proteins and modified protein materials as therapeutic agents has increased significantly in recent years as a result of the increased understanding of the function of these proteins and because of the advancement of the technologies for their isolation, large scale preparation, and characterization. Many proteins do not have sufficient serum stability to be an effective drug, and the exogenous proteins are found to possess immunogenicity. To circumvent these problems proteins have been modified for therapeutic use. One approach to modification involves the covalent attachment of water-soluble poly-

Address reprint requests to Swapan K. Chowdhury, Sanofi-Winthrop Inc., 1250 South Collegeville Road, Collegeville, PA 19426-0900. mers such as polyethylene glycol (PEG) to the protein molecules. It has been shown that the attachment of polyethylene glycols to proteins (1) reduces, modifies, and in some cases eliminates the immunogenicity of immunogenic proteins, (2) extends the in vivo circulation lifetime, (3) enhances the solubility, (4) increases the stability of proteins by reduction of the tendency for aggregation, and (5) increases their potency, which facilitate more effective use as drugs [1-9]. These advantages of protein modification by polymer attachment have been discussed for a number of proteins that include catalase [3], interleukin-2 [4], bovine serum albumin [5], superoxide dismutase [6], horseradish peroxidase [7], streptokinase [8], asparaginase [9], hirudin [10], adenosine deaminase [11], and stem cell factor [12]. In addition, polyethylene glycol-conjugated 
liposomes have been investigated as a target for the treatment of solid tumors [13].

Conjugation of polymers with proteins produces a heterogeneous mixture of species with a distribution of molecular weights. The heterogeneity originates from the different probabilities of attachments at different locations in the protein as well as the polydispersity of the polymer. Two types of heterogeneity arise from the differences in the attachment probabilities:

1. The attachment of polymers results in a different number of polymers per mole of protein.

2. For the same number of attached polymer groups there are many different possibilities for the attachment, which results in different molecular structures.

The polymers are usually covalently attached to proteins through the $\varepsilon$-amino groups of lysine residues [2-12], although linkings also have been made via thioethers of cysteine residues [14]. The conditions that are typically used for the PEG-attachment to proteins result in the incorporation of a different number of PEG molecules per mole of protein. The potency of protein-polymer complexes may depend on the extent of conjugation, which must therefore be established before the material can be considered as a potential drug. As a result, the characterization of polymerprotein complexes has generated a lot of interest in recent years.

A number of techniques reported for the characterization of protein-polymer conjugates include classical protein characterization methods, such as chromatography, capillary electrophoresis, light scattering, isoelectric focusing, polyacrylamide gel electrophoresis $[6 a, 6 b, 10,15,16]$, and mass spectrometry $[12 b, 17,18]$. The classical techniques do not provide sufficient resolution to resolve all of the protein species that contain different numbers of polymer units. Vestling et al. [17, 18] recently described a method for the characterization of PEG-conjugated superoxide dismutase (SOD) using mass spectrometry. In this procedure, the PEGs are removed from the protein by treatment with a base and the protein is left with a succinyl marker at the site of attachment. The PEG-deconjugated protein is then subjected to peptide mapping by using conventional and tandem mass spectrometry for the identification of the site of attachment by identification of the site of the marker and determination of the amount of PEG attached to each site by isotope labeling experiments [18]. This method provides a measurement of the extent of protein-PEG-conjugation at different sites. Unfortunately, the method involves complicated and time-consuming mass spectrometry operations that cannot be applied easily for routine determinations of sample stabilities. More recently, Watson et al. [12b] used matrix-assisted laser desorption-ionization (MALDI) mass spectrometry [19] to obtain a spectrum of stem cell factor coupled with PEG molecules. The mass spectrum showed resolved peaks of PEG-conjugated stem cell factor that correspond to 1, 2, 3, and 4 PEGs attached to stem cell factor [12b]. However, a detailed account was not given of the reproducibility of this technique for the determination of PEG-conjugated proteins, detection limit, and how the MALDI data compared with data from other measurements.

In the present report we describe the utility of matrix-assisted laser desorption-ionization (MALDI) mass spectrometry as a method for the rapid determination of (1) product heterogeneity that results from the different number of PEG molecules attached, (2) comparison with the data obtained from other methods, and (3) its ease of use for the determination of lot-to-lot variation for quality control and stability testing of PEG-conjugated SOD. Furthermore, the reproducibility of measurements of PEG-conjugated SOD by MALDI was investigated.

Superoxide dismutase is an important enzyme that plays a vital role in human physiology. It catalyzes the destruction of the $\mathrm{O}_{2}^{-}$free radical (eq 1):

$$
2 \mathrm{O}_{2}^{-}+2 \mathrm{H}^{+} \rightarrow \mathrm{O}_{2}+\mathrm{H}_{2} \mathrm{O}_{2}
$$

Superoxide dismutase protects oxygen-metabolizing cells against harmful effects (such as aging, lipid peroxidation, and peroxidative hemolysis of red blood cells) of superoxide free radicals [20] and hyaluronate against depolymerization by free radicals. Other therapeutic utilities of SOD and PEG-conjugated SOD include antiinflammatory effects [21] and treatment for injuries [22]. The PEG-conjugated bovine superoxide dismutase (pegorgotein) ${ }^{1}$ samples used in the present investigation were synthesized by the reaction of bovine SOD with PEG reagents [6c] of approximate molecular weight of 5000 [16-18]. The bovine SOD in its native form consists of two identical subunits, each of which contains 151 amino acids that include 10 lysines and an acetylated amino terminus [23]. Because lysines were targeted for the attachment of PEG to SOD, there are 10 possible sites of attachment per monomer. We describe a method that provides rapid fingerprinting of PEG-conjugated SOD samples that determines the number of PEGs attached to SOD and quantitates the amounts of PEG-conjugated SOD each with a different number of PEG units. Data presented here originate from the measurements of eight different batches of PEG-conjugated SOD samples prepared separately with different preparations of PEG, of different lifetime, and stored under different experimental conditions.

\footnotetext{
${ }^{1}$ Superoxide dismutase from bovine origin as $\mathrm{Cu}-\mathrm{Zn}$ mixed chelate with enzyme activity is also known as orgotein (United States Pharmacopeial Convention, Inc., C. A. Fleeger, Ed., 1993, pp 462). Hence the PEG-conjugated bovine SOD is called pegorgotein. The Sanofi Winthrop, Inc. proprietary name of pegorgotein is Dismutec ${ }^{\mathrm{TM}}$. Pegorgotein also has been mentioned as PEG-SOD in the literature.
} 


\section{Experimental}

The PEG-conjugated SOD samples used in the present investigation were prepared via the procedure [9a] discussed elsewhere [16-18]. Briefly, polyethylene glycol of approximate molecular weight 5000 was activated by conversion of the free hydroxyl group at one end of the polymer to a succinylsuccinimide; the other end was capped with a methoxyl group. The activated PEG was then converted to PEG-conjugated SOD by reaction with bovine SOD at $\mathrm{pH} 7.8$. The activated PEG reacts with the primary amines of SOD [9a, 16-18]. Eight batches of PEG-conjugated SOD samples ( $\$ 1$ through S8 studied here) differ from each other in their lifetime, type of PEG used to prepare them, storage temperature, and so forth. These parameters are given in Table 1. The polydispersity of one batch of PEG examined was 1.04 .

The MALDI data were acquired on a Finnigan-MAT Vision-2000 time-of-flight mass spectrometer (Bremen, Germany) [24] equipped with an ion reflectron system. The experiments were carried out at Charles Evans and Associates (Redwood City, CA). All the data presented here were obtained in the reflectron mode of operation. Laser radiation of 337-nm wavelength generated by a $\mathrm{N}_{2}$ laser (Laser Science Inc., Newton, MA) at 3-ns pulse width was used to produce ions of PEG-conjugated SOD from a stainless steel target on which a sample solution was codeposited with that of a matrix in equal volumes ( $0.5 \mu \mathrm{L}$ each). Sample solutions were prepared by dilution of the original PEGconjugated SOD solution $(\sim 8 \mathrm{mg} / \mathrm{mL})$ in distilled water (Fluka, Ronkonkoma, NY) to a concentration of $20 \mu \mathrm{M}$, assuming an average molecular weight of 36,000 . The matrix solution was prepared in two steps [25]. In the first step, a $10-\mathrm{mg} / \mathrm{mL}$ solution of 2,5-dihydroxy benzoic acid (Aldrich Chemical Co., Milwaukee, WI; $99 \%$ pure) was prepared in $65 \%$ of $0.1 \%$ aqueous trifluoroacetic acid (Spectrum Chemicals, Los Angeles, $\mathrm{CA}$ ) and $35 \%$ acetonitrile (high-performance liquid chromatography grade; Sigma Chemical Co., St. Louis, $\mathrm{MO}$ ) and a solution of 2-methoxy,5-hydroxy benzoic acid (Aldrich; $99 \%$ pure) of the same concentration was prepared in equal volumes of water and ethanol. In the second step, these two matrix solutions were mixed at a ratio of 9:1 v/v, respectively, to obtain the final matrix solution that was codeposited on the probe tip with the sample solution. After the sample-matrix solution dried, the probe tip was inserted into the vacuum of the mass spectrometer for MALDI. Data from 100 laser shots were summed to obtain the final spectrum. The mass-to-charge ratio scale of the data was calibrated by using protonated ions of the monomer and dimer of SOD. The area of the ion peaks was deduced by using the Finnigan-MAT data system with a manual assignment of the horizontal baseline for individual peaks. The ions initially were accelerated from the sample target with a translational energy of $3 \mathrm{keV}$ and postaccelerated by another $20 \mathrm{keV}$ prior to detection.

\section{Results and Discussion}

\section{Mass Spectra of Polyethylene Glycol-Conjugated Superoxide Dismutase}

A MALDI spectrum of the standard batch (S1) of PEG-conjugated SOD sample is shown in Figure 1. The spectrum shows the presence of eight peaks designated with numerals $0-7$ with $\mathrm{m} / z$ values of 15,603 , $20,745,25,995,31,093,36,265,41,496,46,648,51,600$, respectively. The number on each peak, shown below the mass-to-charge ratio values, corresponds to the number of PEG groups present in the PEG-conjugated SOD molecules. As mentioned previously, PEGconjugated SOD in solution consists of two noncovalently attached subunits of SOD backbone attached to PEG. Each SOD molecule with an acetylated amino terminus has 10 lysines to which PEG molecules can attach. During the MALDI the two noncovalently held

Table 1. Information on the lifetime, storage temperature, PEG, and preparation of eight batches of PEG-conjugated SOD examined here

\begin{tabular}{llccr}
\hline $\begin{array}{l}\text { Sample } \\
\text { i.d. }\end{array}$ & $\begin{array}{c}\text { Activated } \\
\text { PEG i.d. }\end{array}$ & $\begin{array}{c}\text { Diol content } \\
\text { of the PEG } \\
(\%)\end{array}$ & $\begin{array}{c}\text { Lifetime } \\
\text { (months) }\end{array}$ & $\begin{array}{c}\text { Storage } \\
\text { temperature } \\
\left({ }^{\circ} \mathrm{C}\right)\end{array}$ \\
\hline \hline S1 & Batch 1 & $\sim 3$ & 15 & -20 \\
S2 & Batch 2 & $\sim 30$ & 56 & 5 \\
S3 & Batch 3 & $\sim 3$ & 36 & 5 \\
S4 & Batch 3 & $\sim 3$ & 36 & 15 \\
S5 & Batch 4 & $\sim 3$ & 36 & 5 \\
S6 & Batch 4 & $\sim 3$ & 36 & 15 \\
S7 & Batch 5 & $\sim 3$ & 12 & 5 \\
S8 & Batch 6 & $\sim 3$ & 6 & 5 \\
\hline
\end{tabular}

\footnotetext{
The diol content of a sample is the amount of PEG that contains two hydroxyl groups. The PEG in the sample is expected to have one hydroxy group in one end and a methoxy group at the other. The hydroxyl group is used to couple to proteins. Thus the diols will have a free hydroxyl group after conjugation with a SOD molecule.
} 


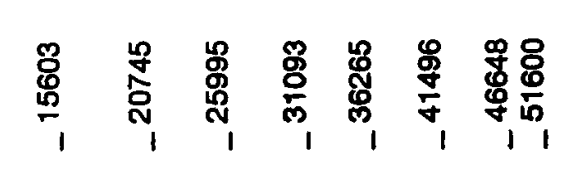

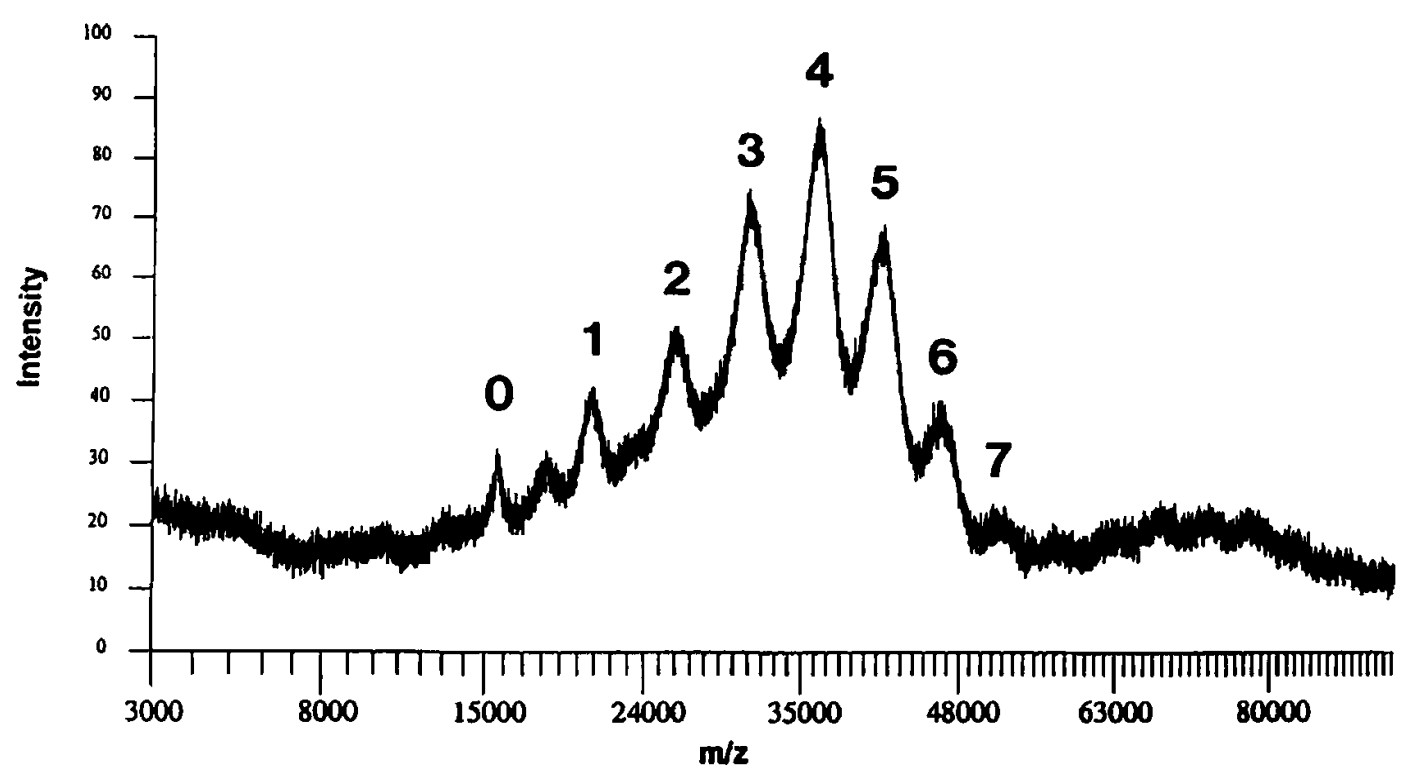

Figure 1. Matrix-assisted laser desorption-ionization spectrum of the PEG-conjugated SOD sample S1. The numbers on the peaks designated 0-7 indicate the number of PEG molecules attached. The numbers at the top of the spectrum are the centroided mass-to-charge ratio values of the corresponding peaks. Approximately $10 \mathrm{pmol}$ of the sample was deposited on the probe tip. The spectrum was obtained by summing the spectra from 100 individual laser shots. Note the square root mass-to-charge ratio scale.

subunits dissociate and a spectrum that corresponds to one PEG-conjugated SOD subunit is observed. In Figure 1 up to seven PEG molecules were identified to be attached to SOD monomers. Each of the adjacent peaks designated $0-7$ is determined to be separated by approximately $5000 \mathrm{u}$. This value agrees with the average molecular weight of PEG used to prepare PEGconjugated SOD. Therefore, these adjacent peaks differ by one PEG unit. The area of each peak provides information on the amount of PEG-conjugated SOD that contains the number of PEG molecules identified by the number in Figure 1. Thus, the spectrum (Figure 1) provides a rapid fingerprinting of PEG-conjugated SOD on the distribution of attached PEG. The massto-charge ratio values shown at the top of the spectrum correspond to the centroids of the peaks. The intensity distribution within any given ion peak in Figure 1 corresponds, in the most part, to the polydispersity of the attached PEG.

The molecular weight of a SOD monomer is 15,592 . Therefore, the peak designated 0 in Figure 1 may be considered to arise from a SOD monomer. However, observation of this peak in the mass spectrum does not necessarily indicate the presence of PEG-free SOD in the PEG-conjugated SOD sample. This peak may originate from two different sources.
1. Those PEG-conjugated SOD molecules (dimers in solution) with PEG on only one of the SOD monomers will produce equimolar amounts of SOD with PEG and SOD without PEG upon dissociation. Therefore, one can expect to see SOD monomer ions in the MALDI spectra even if the PEG-conjugated SOD sample contains no PEG-free SOD.

2. The mass-to-charge ratio value of the peak designated $0(15,603)$ is approximately half of that of the peak designated $3(\mathrm{~m} / z$ 31,093). Thus, a small doubly charged ion contribution of PEG-conjugated SOD with three PEG molecules will appear at the peak designated 0 .

There is also a third possibility that may contribute to that peak; PEG-conjugated SOD molecules that would undergo decomposition during MALDI with the elimination of attached PEGs (covalent dissociation) will occur also at peak 0 . However, the data presented below provide evidence to support the absence of any significant on-target (prompt) fragmentation of PEGconjugated SOD under the present MALDI experimental conditions. All the data presented here were acquired with near threshold laser fluence required to produce ion desorption from PEG-conjugated SOD. 
We have investigated the contribution of ions with two charges. As shown in Figure 1, the ion peak $(\mathrm{m} / \mathrm{z}$ $18,153)$ located between the peaks designated 0 and 1 most likely arises from a PEG-conjugated SOD molecule that contains four PEG per monomer with two charges. This PEG-conjugated SOD ion peak with two charges is free of interference from other ions and was used to determine the $2+$ ion contribution of PEG-conjugated SOD from 10 different sets of data points. In addition, data from two other peaks also were used to deduce the $2+$ ion contribution. The amount of PEG-conjugated SOD ions with two charges was calculated from the spectra to be $16 \pm 4 \%$ relative to that of PEG-conjugated SOD ion with only one charge, where both had the same number of PEG groups. The peak 0 in Figure 1 is only $3.2 \%$ of the total PEG-conjugated SOD contribution, whereas peak 3 is $19.3 \%$. The calculated $2+$ ion contribution in peak 0 is $3.1 \%$ ( $16 \%$ of 19.3 is 3.1 ). In other words, the ion abundance in peak 0 (Figure 1 ) is very close to that which can be expected from the $2+$ ion contribution of PEG-conjugated SOD with three PEGs (assuming a similar $2+$ ion contribution of PEG-conjugated SOD molecules that contain three and four PEG groups). This observation suggests the absence of PEG-free SOD in the PEG-conjugated SOD sample and is in agreement with the detection of no free SOD molecules in gel permeation chromatographic measurement of the sample S1. Furthermore, the observation of only $3.2 \%$ abundance of peak 0 eliminates the possibility of prompt fragmentation of PEG-conjugated SOD ions to SOD ions in the present MALDI experimental conditions. In addition, the peakwidth at half-maximum of the peak designated 0 in Figure 1 is larger than that which is typically observed for the $1+$ ion of SOD and is approximately half the width of the peak designated 3 . The $2+$ ion contribution observed by Watson et al. [12b] in the MALDI spectra of PEG-conjugated stem cell factor, however, appears to be much higher than that observed for PEG-conjugated SOD in the present investigation (Figure 1). This difference in the $2+$ ion contribution could be due to, among others, the different protein backbones (stem cell factor versus SOD) involved and/or the different matrix materials used. Watson et al. [12b] used sinapinic acid as the matrix, whereas a mixture of 2,5-dihydroxy benzoic acid and 2-hydroxy,5-methoxy benzoic acid [25] was used in the present studies. The smaller $2+$ ion contribution observed in the present study reduces the spectral complexity and is particularly important in the case of spectra of PEG-conjugated SOD in which $2+$ ion peaks unfortunately overlap with some of the peaks from $1+$ ions.

\section{Reproducibility of Measurements and Detection Limit}

To establish whether the technique can be used to fingerprint PEG-conjugated SOD for the determination of the extent of PEG-conjugation, the reproducibility in the MALDI measurements of PEG-conjugated SOD samples was investigated. The standard sample $\mathrm{S} 1$ was prepared separately, examined by the MALDI method nine times on three different dates, and measured between other batches of samples. The intensity values were deduced from the area of each peak that corresponded to the different number of PEG groups (see Figure 1). The average data of nine measurements together with the standard deviation of the data points are shown in Figure 2. The standard deviation of ion abundances is typically $7-15 \%$ for the abundant peaks, which are at least $13 \%$ of the total ion abundance, whereas the weaker peaks gave a larger deviation (Table 2). The higher standard deviation for weaker peaks (less than $10 \%$ of the total abundance) arises from the higher uncertainties involved in the determination of the area. The standard deviation obtained in three separate measurements of the sample S2, however, is much smaller (Table 2).

The results discussed in the preceding text indicate that the MALDI technique could be used to determine lot-to-lot variability for quality control and stability testing of polymer-conjugated proteins. The intensities that correspond to various number of PEG groups attached, obtained from the MALDI measurements of four samples S1, S2, S7, and S8, are compared in Table 2. The data on sample $S 1$ are an average of nine measurements, those on S2 are an average of three measurements, whereas those on samples S7 and S8 come from single measurements. A comparison of the data obtained from samples S1 and S2 presented in Table 2 shows a clear difference in the extent of PEG attachment in the two samples.

1. The peak that apparently corresponds to zero number of PEG attached per monomer of SOD is much higher in sample S2 compared to that in S1. As previously discussed, the intensity that corresponds to PEG 0 in S1 is close to that which can be expected from the $2+$ ion contribution of PEG-conjugated SOD that contains three PEGs. The $2+$ ion contribution of the PEG-conjugated SOD that contains three PEGs in S2 is expected to be $\sim 3.2 \%$. This value is only one third of what is measured for peak 0 (Table 2). This higher value for the PEG-conjugated SOD monomer ion free of PEG indicates the presence of some PEG-free SOD in sample S2.

2. The intensity of ions of PEG-conjugated SOD that contains a higher number of PEG molecules is higher in the sample S1 compared to that in S2. Thus, for example, in S1 PEG-conjugated SOD ions with four and five PEGs are the most intense whereas in S2 PEG-conjugated SOD ions of 1,2 , and 3 PEGs are the most intense (Table 2). The PEG used to synthesize PEG-conjugated SOD sample S2 contained a higher proportion of diols $(\sim 30 \%)$ compared to only $3 \%$ in the PEG used to make samples S1 and S3-S8. Furthermore, sample S2 is $\sim 5$ years old and 


\section{Average Intensity Distribution of PEG-SOD (per monomer of SOD)}

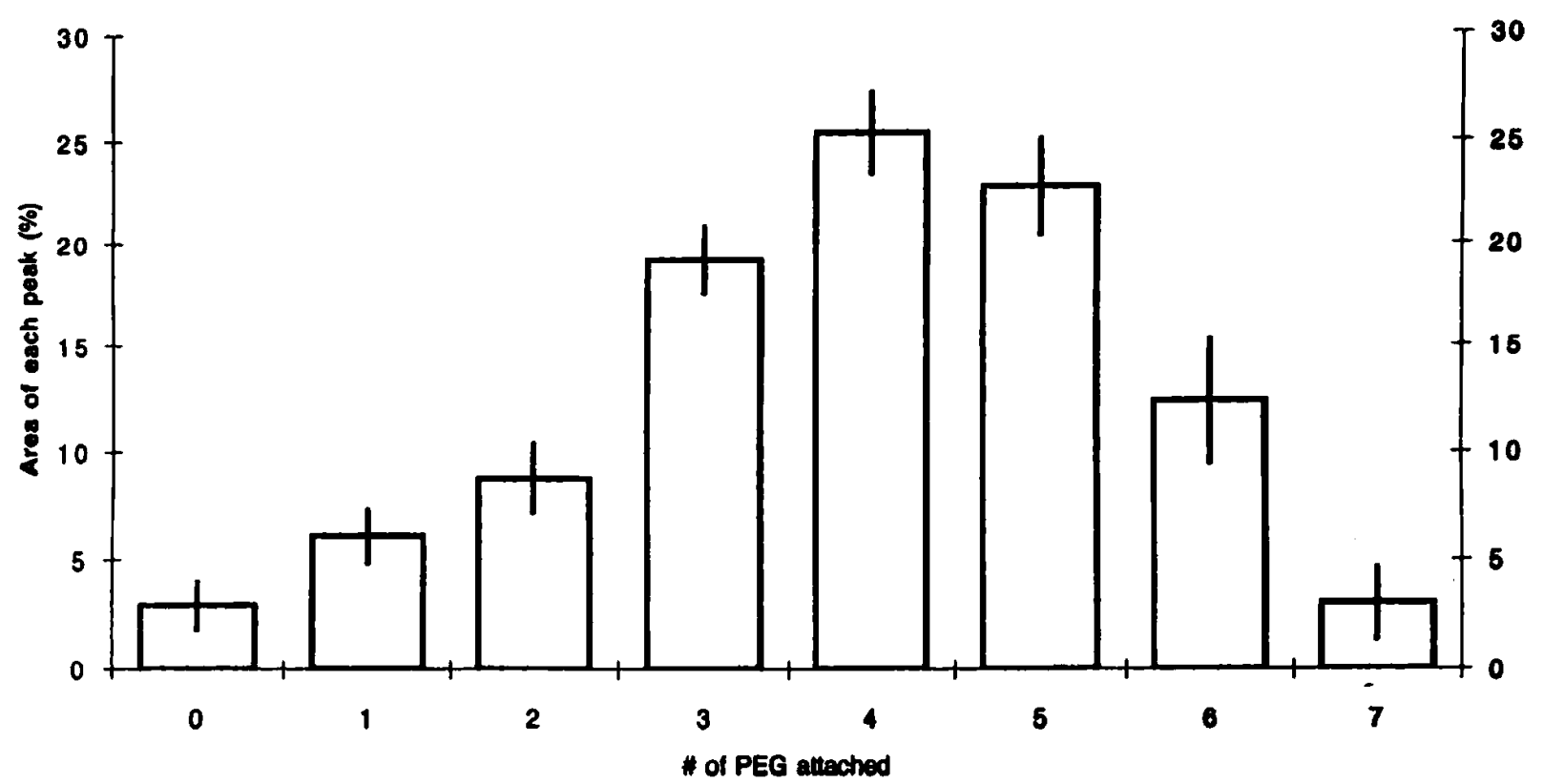

Figure 2. The average and standard deviation values of the area of different ion peaks of PEG-conjugated SOD obtained from nine separate MALDI measurements. The bars designated 0-7 correspond to PEG-conjugated SOD ion peaks that contain 0-7 PEG molecules, respectively. The standard deviation observed in the measurements of each peak is shown by the vertical line on top of the bar that represents the peak. The measurement conditions were the same as those given for Figure 1.

substantial PEG-deconjugation would be expected to have occurred. MALDI data clearly demonstrate that there are differences in the PEG content in the two samples.

Samples S7 and S8 were prepared from the same batch of PEG that was used to prepare sample S1 (Table 2). These samples differ only in their age and storage conditions. Sample $S 1$ is 15 months old and was stored at $-20^{\circ} \mathrm{C}$, whereas samples $\mathrm{S} 7$ and $\mathrm{S} 8$ are, respectively, 12 and 6 months old; both were stored at $5^{\circ} \mathrm{C}$. The similarity in the mass spectra of $\mathrm{S1}, \mathrm{S7}$, and S8 (Table 2) suggests that at the storage temperature of $5{ }^{\circ} \mathrm{C}$, the PEG-conjugated SOD samples do not degrade to a measurable extent within the 12-month duration examined here.

Table 2. Comparison of the intensities of various ion peaks that contain different numbers of PEG molecules attached per monomer of SOD observed in the MALDI spectra of four different batches of PEG-conjugated SOD samples ${ }^{\mathrm{a}}$

\begin{tabular}{lcccc}
\hline $\begin{array}{l}\text { Number of } \\
\text { PEG attached }\end{array}$ & Sample S1 $^{\mathrm{b}}$ & Sample S2 $^{\mathrm{c}}$ & Sample S7 $^{\mathrm{d}}$ & Sample S8 $^{\mathrm{d}}$ \\
\hline \hline 0 & $2.9 \pm 1.1$ & $9.6 \pm 1.5$ & 4.9 & 4.9 \\
1 & $6.1 \pm 1.2$ & $19.2 \pm 2.7$ & 4.9 & 5.2 \\
2 & $8.8 \pm 1.6$ & $20.0 \pm 2.2$ & 6.9 & 8.1 \\
3 & $19.2 \pm 1.6$ & $20.2 \pm 2.2$ & 12.6 & 13.4 \\
4. & $25.4 \pm 1.9$ & $15.0 \pm 2.1$ & 21.5 & 20.4 \\
5 & $22.8 \pm 2.3$ & $10.1 \pm 0.5$ & 26.3 & 26.7 \\
6 & $12.4 \pm 2.9$ & $4.9 \pm 0.7$ & 16.6 & 13.4 \\
7 & $3.0 \pm 1.6$ & - & 4.9 & 7.6 \\
8 & - & - & 1.6 & 1.2 \\
\hline
\end{tabular}

"Intensity values are obtained from the area of each peak in the MALDI mass spectra, converted into percent of total intensities. No correction for $2+$ ion contribution is taken into account.

Data from nine different measurements.

CData from three different measurements.

Data from a single measurement. 
All the MALDI data discussed so far were acquired from 20- $\mu \mathrm{M}$ sample solutions (approximate $\mathrm{MW}=$ $36,000)$ with $10 \mathrm{pmol}(0.5 \mu \mathrm{L})$ deposited on the probe (Figure 1, for example). Figure 3 shows PEGconjugated SOD spectra of sample S1 obtained from 1(top) and 0.1-pmol (bottom) materials. The spectrum from 1-pmol sample is quite similar to that obtained from 10-pmol sample (Figure 1), although the former has a lower signal-to-noise ratio. When the sample amount was reduced to $0.1 \mathrm{pmol}$, the spectrum lacked any discernible information (Figure 3). These experiments suggest that at least $1 \mathrm{pmol}$ of sample may be needed to obtain an informative spectrum of PEGconjugated SOD. In homogeneous samples, such as SOD, the amount deposited on the probe corresponds to a single species. In contrast, in PEG-conjugated SOD 1 pmol represented thousands of different species with different molecular weight. These "thousands of different species" come from the number of lysines times the PEG molecular weight distribution times the population probability per site. As a result, the concentration of a given species in the PEG-conjugated SOD sample is much lower than has been described. Larger quantities of heterogeneous samples, as such, are needed to obtain a MALDI spectrum even though the detection limit may be as good as those for proteins.

\section{Determination of the Stability of \\ Polyethylene Glycol-Conjugated \\ Superoxidase Dismutase Samples}

Because of good reproducibility of measurements, ease of use, rapidity of analyses, and relatively straightforward data interpretation, MALDI can be used rou-

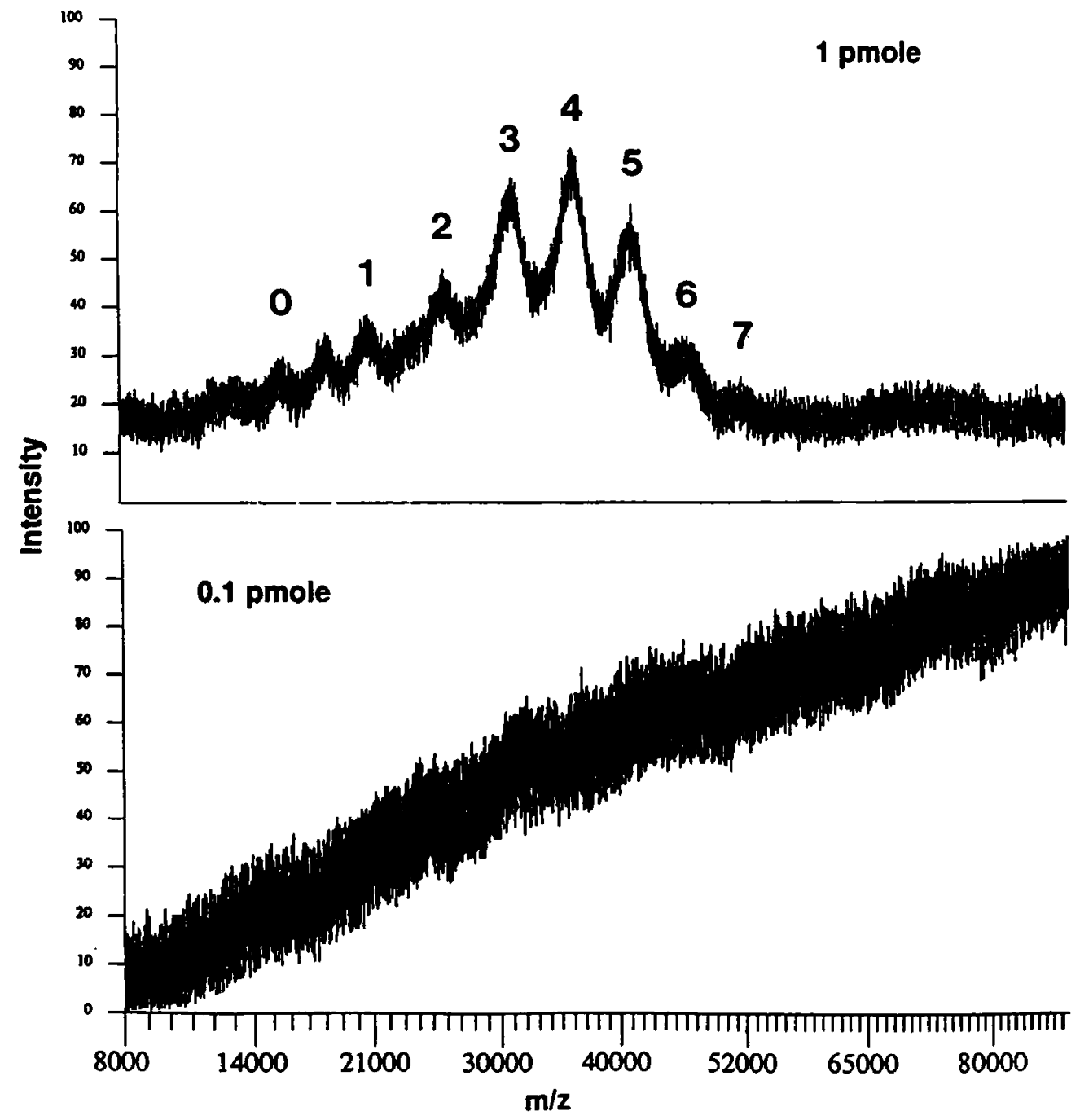

Figure 3. MALDI mass spectra of PEG-conjugated SOD sample S1. The top spectrum was obtained from $1.0 \mathrm{pmol}$ and the bottom spectrum from $0.1 \mathrm{pmol}$ of sample deposited on the probe. The experimental conditions were the same as those used in acquisition of the spectrum shown in Figure 1. The top spectrum is similar to that in Figure 1 (10 pmol), but is of much reduced spectral quality (signal to noise). The bottom spectrum does not provide any information on the PEG-conjugated SOD sample. These data indicate that at least $1 \mathrm{pmol}$ of PEG-conjugated SOD may be needed to obtain a meaningful MALDI spectrum. 
tinely to determine the stability of polymer-conjugated proteins. Figure 4 shows the intensity of PEGconjugated SOD ions plotted against different number of PEG groups attached for four different samples-S3, $\mathrm{S4}, \mathrm{S} 5$, and $\mathrm{S6}$. S3 and $\mathrm{S4}$ are the same material stored at 5 and $15^{\circ} \mathrm{C}$, respectively, for 36 months. Similarly, S5 and S6 arise from the same batch of PEG-conjugated SOD and were stored at 5 and $15^{\circ} \mathrm{C}$, respectively, for the same duration (Table 1). Examination of the data presented in Figure 4 demonstrates that in the MALDI spectra of samples stored at $15^{\circ} \mathrm{C}$, the intensity distribution of ions corresponds to a PEG distribution with fewer PEGs than those for samples stored at $5{ }^{\circ} \mathrm{C}$. For example, the peak that apparently corresponds to 0 PEG is about 5\% in S3, whereas it is higher than $10 \%$ in S4, and the ion peak that contains four PEGs is $\sim 23 \%$ in S3 and has been reduced to $\sim 4 \%$ in S4. A similar ion intensity trend also was observed in the MALDI spectra of samples S5 and S6. That is, PEGconjugated SOD molecules in the samples stored at 5 ${ }^{\circ} \mathrm{C}$ contain a higher number of PEG molecules per mole of SOD than those stored at $15{ }^{\circ} \mathrm{C}$. Because there is no difference in the experimental conditions used to obtain the spectra, the lower PEG content in S4 and S6 most likely arises from the PEG-deconjugation of these samples at a higher temperature. Samples S4 and S6 may even contain PEG-free SOD (Figure 4). This observation was confirmed by the detection of PEG-free SOD in the gel permeation chromatographic determination of the sample. These results clearly demonstrate the utility of the technique to examine the stability of the samples for quality control of polymer-attached proteins.

\section{Comparison of Matrix-Assisted Laser Desorption-Ionizaton Data with Those Obtained from Other Techniques}

To determine quantitative aspects of the MALDI technique for the characterization of polymer-conjugated proteins, the data obtained from the peptide mapping method by using liquid chromatographic quantitation [26] were compared with the data obtained from the MALDI analysis. Sample S1 was examined by both techniques. In the peptide mapping procedure, the PEG-conjugated SOD sample is cleaved into small peptides by treatment with Asp-N, an enzyme that cleaves at the amino terminus of the acidic residues. The peptides that contained nonmodified lysines were quantitated from the liquid chromatogram and the data were compared with those from the same peptide that originated from SOD. The measurements provide an estimation of the extent of PEGation at each lysine. Data obtained for various lysines in the sample $S 1$ are as follows: Lys 3, 45\%; Lys 9, 60\%; Lys-23, not determined; Lys 67, 68, 73, 91\%; Lys $89,66 \%$; Lys 120 , $100 \%$; Lys $134,45 \%$; Lys $151,69 \%$. The amount of PEG-conjugation at Lys 23 could not be determined because the peptide that contained this lysine was not detected and Lys 67, 68, and 73 were measured together as they appeared in one peptide. For Lys 23 , for which no peptide mapping data were available, we have used data (54\% PEG-conjugation) reported by Vestling et al. [18] for a different batch of sample, and for Lys 67,68 , and 73 we have assumed equal contribution ( $30 \%$ each) from the three attachment sites. These values were used as statistical probabilities $P_{i}$

\section{Effect of Temperature on the Stability of PEG-SOD samples}

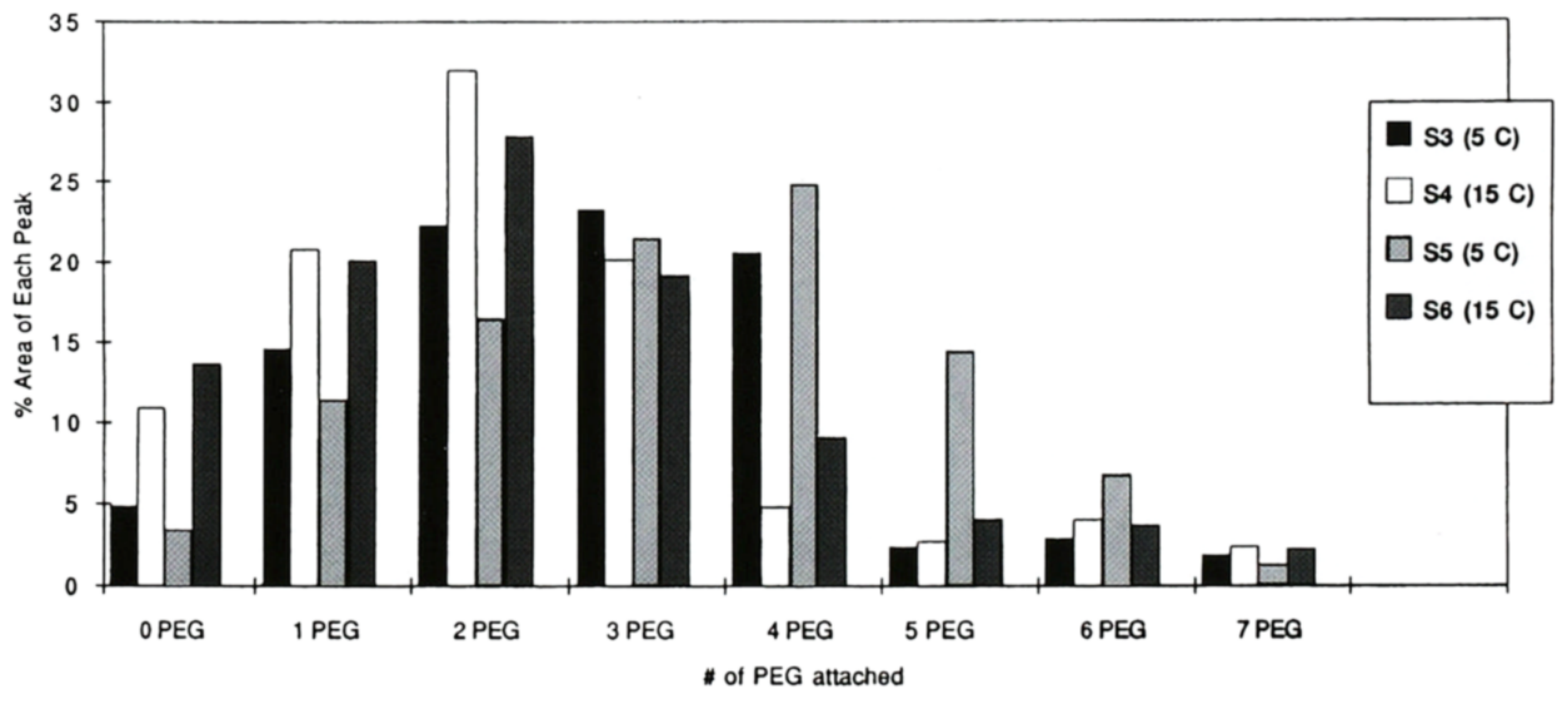

Figure 4. Comparison of the MALDI data of samples S3, S4, S5, and S6. Samples S3 and S4 are the same material but stored at 5 and $15{ }^{\circ} \mathrm{C}$, respectively, for 36 months. $\mathrm{S} 5$ and $\mathrm{S} 6$ arise from the same material (but different than S3 and $\mathrm{S} 4$ ) stored at 5 and $15^{\circ} \mathrm{C}$, respectively, for 36 months. S4 and S6 samples show a much reduced extent of PEG-conjugation compared to S3 and S5. 
for PEG-conjugation at these sites. Thus $P_{1}=0.45$, $P_{2}=0.6, \quad P_{3}=0.54, \quad P_{4}=P_{5}=P_{6}=0.3, \quad P_{7}=0.66$, $P_{8}=0.99, P_{9}=0.45$, and $P_{10}=0.69$.

If $P_{i}$ is the probability of the PEG-conjugation at the $i$ th site, then the probability of PEG-conjugated SOD molecules that contain different numbers of attached PEG groups can be expressed by formula (2):

$$
\prod_{i=1}^{10} P_{i}^{\sigma_{i}}\left(1-P_{i}\right)^{\left(1-\sigma_{i}\right)}
$$

where $\sigma_{i}$ defines the status of the site $i$. If the site $i$ is PEG-conjugated, then $\sigma_{i}=1$; if the site is unoccupied, $\sigma_{i}=0$. For example, if we have only two sites with attachment probabilities $P_{1}(0.45)$ and $P_{2}(0.6)$, formula (2) reduces to formula (3):

$$
P_{1}^{\sigma_{1}}\left(1-P_{1}\right)^{\left(1-\sigma_{1}\right)} \times P_{2}^{\sigma_{2}}\left(1-P_{2}\right)^{\left(1-\sigma_{2}\right)}
$$

From formula (3) the probability of molecules with no PEG attached is $0.55 \times 0.4=0.22\left(\sigma_{1}=\sigma_{2}=0\right)$, that for one site occupied is $0.45 \times 0.4\left(\sigma_{1}=1, \sigma_{2}=0\right)$ $+0.55 \times 0.6\left(\sigma_{1}=0, \sigma_{2}=1\right)=0.51$, and that for both sites occupied is $0.45 \times 0.6=0.27\left(\sigma_{1}=1, \sigma_{2}=1\right)$. These values are expected from the statistical distribution of the attachment of PEGs on the two sites with probabilities 0.45 and 0.6 .

The number of possibilities, however, increases beyond the ability for manual calculation when the maximum value of $i$ becomes equal to 10 . Therefore, based on the formula (2) a computer algorithm was developed to calculate the probability of PEG-con- jugated SOD molecules that contain different numbers of attached PEG groups. The values obtained, based on the peptide mapping data, for 0-10 attached PEG groups; respectively (in percent), are $0,0.2,2.0,8.3$, 19.0, 26.8, 24.1, 13.8, 4.9, 1.0, and 0. A comparison of these values with those obtained from the MALDI data (Table 2, S1), after correction for $2+$ ion contributions, is given in Figure 5. The two curves that represent data from the two sources are strikingly similar except that the peptide mapping values predict, for the same abundance, one more PEG per SOD monomer. There are a number of possibilities for the origin of this difference.

1. There are uncertainties involved in the determination of the probabilities of attachments by peptide mapping. The probability $P_{3}$ was obtained from a different type of measurement performed on a different batch of sample [18] and $P_{4}, P_{5}$, and $P_{6}$ were estimates from the total value for the three.

2. The discrimination in the desorption and ionization of this class of materials in the MALDI is unknown. No attempt was made to determine the massto-charge ratio-dependent detection efficiency of polymer-conjugated proteins in the present study. A uniform MALDI response independent of molecular weight was assumed in the data reported here.

With all these possible sources of uncertainties, the agreement between the two methods is certainly good. The preliminary results presented here indicate that

\section{Comparison of data from MALDI and peptide mapplng}

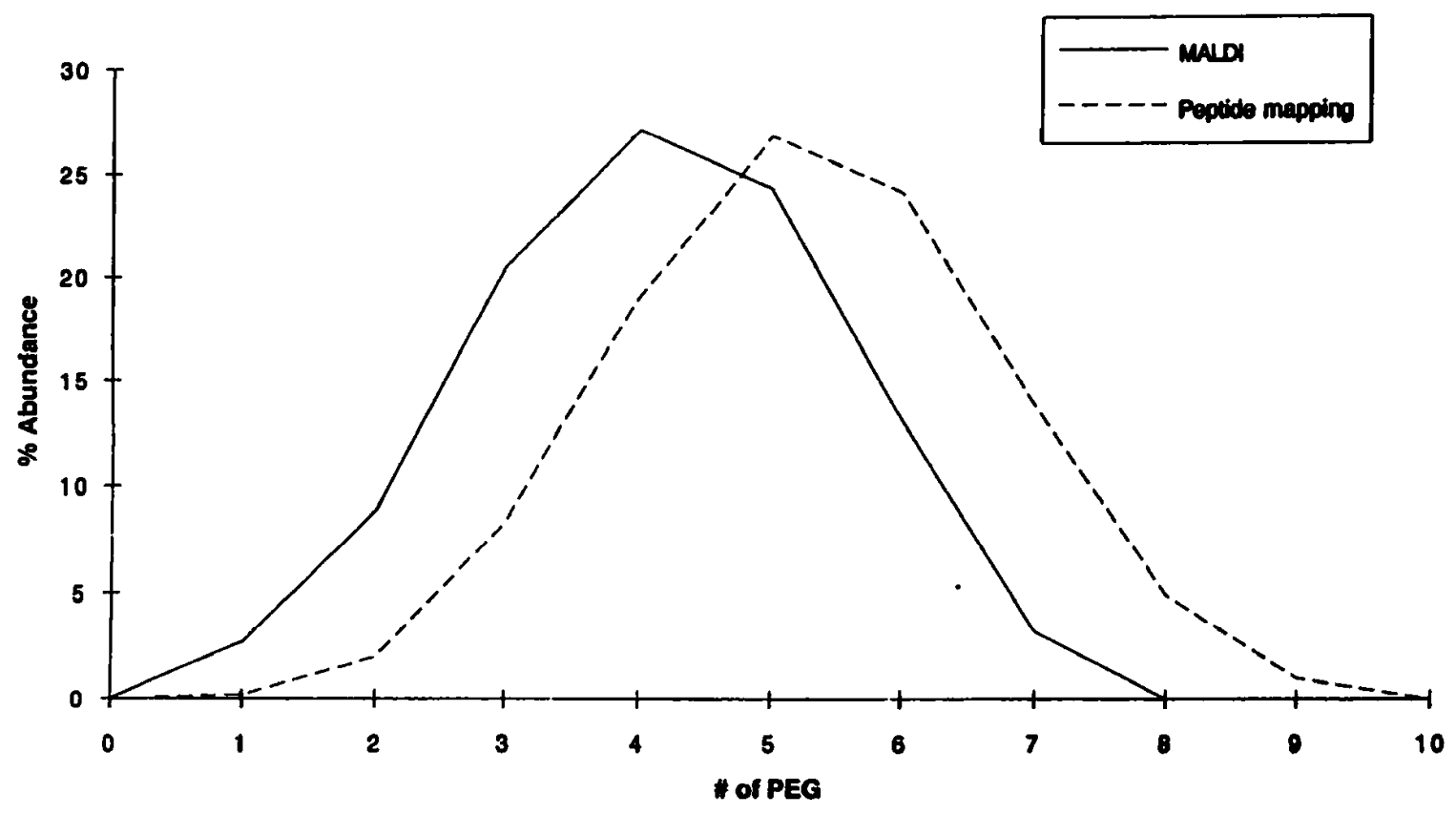

Figure 5. Comparison of the amount (percent of the total) of PEG-conjugated SOD that contains different number of PEG groups determined by MALDI with those statistically deduced from the percent PEG-conjugation values for each attachment site measured by the peptide mapping method. 
MALDI may be used to quantitatively determine PEG-conjugated proteins within the accuracy observed here. Further investigations are underway to increase our understanding of the effect of various factors that govern the desorption-ionization of polymerconjugated proteins in MALDI. The high sensitivity, rapidity in analyses, relatively straightforward data interpretation, and good reproducibility in measurements make MALDI a viable analytical tool for fingerprinting PEG-conjugated SOD as well as other polymer-conjugated proteins [12b].

\section{Acknowledgment}

The authors thank R. C. Khan-Malek for writing the computer algorithm used to calculate the extent of PEG-conjugation from the attachment probabilities, J. Chakel of Charles Evans and Associates for the MALDI measurements, and J. Strasters, J. Bullock, and R. Weinkam for their valuable suggestions.

\section{References}

1. Inada, Y.; Matsushima, A.; Kodera, Y.; Nishimura, H. J. Bioact. Compat. Polym. 1990, 5, 343-364.

2. Abuchowski, A.; Davis, F. F. In Enzymes As Drugs; Holcenberg, I. S.; Roberts, J., Eds.; Wiley-Interscience, New York, 1981; pp 367-383.

3. Abuchowski, A.; McCoy, J. R.; Palczuk, N. C.; van Es, T.; Davis, F. F. J. Biol. Chem. 1977, 252, 3578-3581.

4. (a) Katre, N. V.; Knauf, M. J.; Laird, W.J. Proc. Natl. Acad. Sci. USA 1987, 84, 223-226, (b) Knauf, M. J.; Bell, D. P.; Hirtzer, P.; Luo, Z.-P.; Young, J. D.; Katre, N. V. J. Biol. Chem. 1988, 269, 15064-15070; (c) Katre, N. V. I. Immunol. 1990, 144, 209-213.

5. Abuchowski, A.; van Es, T.; Palczuk, N. C.; Davis, F. F. J. Biol. Chem. 1977, 252, 3578-3581.

6. (a) Veronese, F. M.; Caliceti, P.; Pastorino, A.; Schiavon, O.; Sartore, L.; Banci, L.; Scolaro, L. M. J. Controlled Release 1989, 10, 145-154; (b) Somack, R.; Saifer, M. G. P.; Williams, L. D. Free Rad. Res. Commun. 1991, 12-13, 553-562; (c) Pyatak, P. S.; Abuchowski, A.; Davis, F. F. Res. Commun. Chem. Pathol. Pharmacol. 1980, 29, 113-127.

7. Wirth, P.; Souppe, J.; Tritsch, D.; Biellmann, J.-F. Bioorg. Chem. 1991, 19, 133-142.

8. Rajagopalan, S.; Gonias, S. L.; Pizzo, S. V. I. Clin. Invest. 1985, $75,413-419$.

9. (a) Abuchowski, A.; Kazo, G. M.; Verhoest, C. R., Jr.; van Es, T.; Kafkewitz, D.; Nucci, M. L.; Vian, A. T.; Davis, F. F. Cancer Biochem. Biophys. 1984, 7, 175-186; (b) Ho, D. H.; Brown, N. S.; Yen, A.; Holmes, Y. R.; Keating, M.; Abuchowski, A.;
Newman, R. A.; Krakoff, I. H. Drug. Metab. Dispos. 1986, 14, 349-352.

10. Kurfust, M. F. Anal. Biochem. 1992, 200, 244-248.

11. Davis, S.; Abuchowski, A.; Park, Y. K.; Davis, F. F. Clin. Exp. Immunol. 1981, 46, 649-652.

12. (a) Zsebo, K. M.; Smith, K. A.; Hartley, C. A.; Birkett, N. C.; Andrews, R. G.; Martin, F. H.; Williams, D. A.; Broudy, V.; McNiece, I. K. In Blood Cell Growth Factors: Their Present and Future Use of Hematology Oncology, Proceedings of the Beijing Symposium; Murphy, M. J., Jr., Ed.; AlphaMed Press: Dayton, OH, 1991; pp 194-203; (b) Watson, E.; Shah, B.; Deprince, R.; Hendren, R. W.; Nelson, R. Biotechnol. 1994, 16, 278-280.

13. Allen, T. M. J. Liposome Res. 1992, 2, 289-305.

14. (a) Goodson, R. J.; Katre, N. V. Biotechnol. 1990, 8, 343-346; (b) Benhar, I.; Wang, Q. C.; FitzGerald, D.; Pastan, I. J. Biol. Chem. 1994, 269, 13398-13404.

15. McGoff, P.; Baziotis, A. C.; Maskiewicz, R. Chem. Pharm. Bull. 1988, 36, 3079-3091.

16. Snider, J.; Neville, C.; Yuan, L.-C.; Bullock, J. J. Chromatogr. 1992, 599, 141-155.

17. Vestling, M. M.; Murphy, C. M.; Fenselau, C.; Dedinas, J.; Doleman, M. S.; Harrsch, P. B.; Kutny, R.; Ladd, D. L.; Olsen, M. A. In Techniqes in Protein Chemistry III;-Angegeletti, R. H., Ed.; Academic Press: New York, 1992; pp 477-485.

18. Vestling, M. M.; Murphy, C. M.; Keller, D. A.; Fenselau, C.; Dedinas, J.; Ladd, D.; Olsen, M. Drug Metab. Dispos. 1993, 21, 911-917.

19. (a) Karas, M.; Hillenkamp, F. Annl. Chem. 1988, 60, 2299; (b) Karas, M.; Bachmann, D.; Bahr, U.; Hillenkamp, F. Int. I. Mass Spectrom. Ion Processes 1987, 78, 53; (c) Hillenkamp, F.; Karas, M.; Beavis, R. C.; Chait, B. T. Annl. Chem. 1991, 63, 1193A.

20. (a) Malmstrom, B. G.; Andreasson, L. E.; Reinhammar, B. In The Enzymes, Vol. XII, Part B; Boyer, P. D., Ed.; Academic Press: New York, 1975; p 533; (b) Fee, J. A.; Teitebaum, H. D. Biochem. Biophys. Res. Commun. 1972, 49, 150.

21. (a) Salin, M. L.; McCord, J. M. J. Clin. Invest. 1975, 56, 1319; (b) Miyata, K.; Nakagawa, Y.; Nakamura, M. Europenn Patent Application EP 210761 A1 870204.

22. (a) Liu, T. H.; Beckman, J. S.; Freeman, B. A.; Hogan, E. L.; Hsu, C. Y. Am. I. Physiol. 1989, 256, H589-H593; (b) Muizelaar, J. P.; Marmarou, A.; Young, H. F.; Choi, S. C.; Wolf, A.; Schneider, R. L.; Kontos, H. A. J. Neurosurg. 1993, 78, 375-382.

23. Steinman, H. M.; Naik, V. R.; Abernethy, J. L.; Hill, R. L. J. Biol. Chem. 1974, 22, 7326-7338.

24. Zambias, R. A.; Boulton, D. A.; Griffin, P. R. Tetrahedron Lett. 1994, 35, 4283-4286.

25. Karas, M.; Ehring, H.; Nordhoff, E.; Stahl, B.; Strupat, K.; Hillenkamp, F.; Grehl, M.; Krebs, B. Org. Mass Spectrom. 1993, 28, 1476-1481.

26. Doleman, M.; Emery, C. Internal Study Report 723, Sterling Winthrop, 1993. 